\title{
Novel Effective Photoinitiators for the Production of Dental Fillings
}

\author{
Monika Topa ${ }^{1^{*}}$, and Joanna Ortyl ${ }^{1,2^{*}}$ \\ ${ }^{1}$ Faculty of Chemical Engineering and Technology, Cracow University of Technology, \\ Warszawska 24, 31-155 Cracow, Poland \\ ${ }^{2}$ Photo HiTech Ltd., Bobrzyńskiego 14, 30-348 Cracow, Poland \\ *monika.topa@doktorant.pk.edu.pl \\ *jortyl@pk.edu.pl
}

\begin{abstract}
Photopolymerization is an environmentally-friendly, non-destructive, safe and solvent-free method. Moreover it guarantees low energy consumption. Therefore the photopolymerization is used in many scientific disciplines, including dentistry for production photocurable dental materials. In this work, the new photoinitiating systems based on camphoroquinone (CQ) and iodonium salts with tosyl anion for initiation of photopolymerization of the acrylates monomers bisphenol A - glycidyl methacrylate (BisGMA) and triethyleneglycol dimethacrylate (TEGDMA) was studied. As a reference, camphorquinone (CQ) and ethyl 4(dimethylamino)benzoate (EDB) photoinitiating system was used.
\end{abstract}

Keywords: Photopolymerization, Photoinitiators, Iodonium salts, Camphoroquinone, Dental Fillings

\section{Introduction}

Photopolymerization is popularity because it is an environmentally friendly and non-destructive method for production of different materials for various industries.

The photopolymerization process is so widespread because it has a number of significant advantages. The primary advantage of the photopolymerization over other methods used for preparation of polymer coatings or composites is its speed. Photo-curable compositions are transformed from a liquid state into a fully cross-linked solid within seconds. The photopolymerization ensures no emission of any volatile solvents to atmosphere. Moreover, photopolymerization is carried out at room temperature, without the use of solvents and with low energy consumption [1-5].

Therefore the photopolymerization is used in many scientific disciplines, e.g. in coatings industry for preparation of coating materials; for automotive and furniture industries, in polygraphic industry for making color imprints on different materials or even in medicine for preparation of polymeric hydrogels. Moreover, the photopolymerization nowadays is used mostly in stomatology for production of new generation photocurable dental materials [6-7].

Most popular materials designated for obtaining dental composites through photopolymerization are (meth)acrylatemonomers (RCB - resin based composites) characterized by high reactivity which form an organic matrix. Usually, organic matrixes based on cross-linking dimethacrylates like 2,2-bis[4-(2-hydroxy-3methacryloxypropoxy)phenyl]propane (Bis-GMA), ethoxylated Bis-GMA (EBPDMA),1,6-bis[2-methacryloyloxyethoxycarbonylamino]-2,4,4trimethylhexane

(UDMA), dodecanedioldimethacrylate (D3MA) or triethyleneglycoldimethacrylate (TEGDMA) are used. BisGMA is very popular for the production of photocurable dental composites. Due to the presence of the aromatic structure of Bisphenol A in the core of the molecule, it ensures low volatility of the composition and a high modulus of cured composite [8-13].

On the other hand, the commonly used photoinitiating system for the radical photopolymerization process for dental application is the system based on camphorquinone / aromatic amine. In the step of generating radicals in the 
photolysis process, amine interacted with the excited camphorquinone molecule. This process involves the transfer of the electron from the amine to the ketone, followed by abstraction of the proton. The radicals initiating the polymerization process are mainly radicals formed from amines (Figure 1).

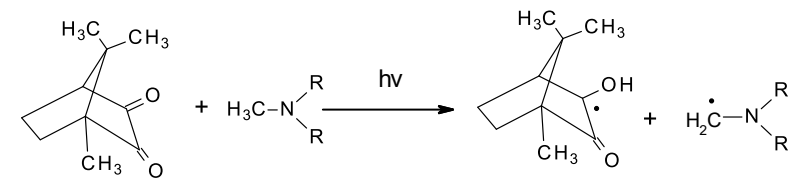

Fig.1. Photolysis of camphorquinone and amine.

The basic problem of this system is the fact that too high concentration of camphorquinone in photocurable dental composites may generate a yellow color. Such discoloration can influence the aesthetics and quality of the final product. In addition, amines are a cytotoxic and genotoxic factor. Therefore, effective photoinitiators for photocurable dental composites without these compounds are needed to be developed [10-9].

In this work, completely new, safe initiating systems based on camphorochinone and a tosyl salt with different substituents have been presented. This system was compared with the standard camphorquinone / amine system. A mixture of BisGMA / TEGDMA monomers in a weight ratio of 7: 3 as the organic matrix was used. It is the most popular organic matrix used to obtain light-cured dental composites. Dental composites conversion rates were studied using real time-FT-IR.

\section{Experimental}

\subsection{Materials}

Bisphenol A-glycidyl methacrylate (BisGMA from Sigma Aldrich) and triethylene glycol dimethacrylate (TEGDMA from Sigma Aldrich) (Figure 2) in the 7:3 weight ratio were applied as a model monomers for free-radical photopolymerization for dental application. Camphorquinone (CQ from Sigma Aldrich) and various tosyl salts (Table 1) were used as new initiation systems for photopolymerization processes. The molar ratio of CQ to the tosyl salt was 2: 1 which corresponds to: 0.5 wt. \% CQ and about 1 wt. tosyl salt. Camphorquinone (CQ from Sigma Aldrich) and ethyl 4- (dimethylamino) benzoate (EDB from Sigma Aldrich) was used as reference in a molar ratio of $2: 1$, which corresponds to $0.5 \mathrm{wt} . \%$. CQ and $0.3 \%$ EDB.

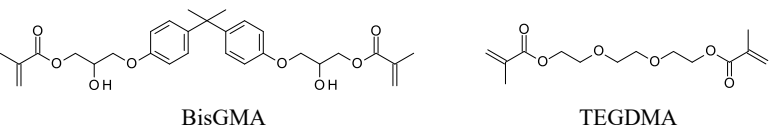

Fig.2. Structures of the monomers.

Table 1. Acronyms, color after curing, and calculated conversion for initiating systems.

\begin{tabular}{|c|c|c|c|}
\hline Acronym & Colour & Substituent & $\begin{array}{c}\text { Conversion } \\
{[\%]}\end{array}$ \\
\hline $\mathrm{CQ}$ & $\bigcirc$ & - & 7,0 \\
\hline $\mathrm{CQ} / \mathrm{EDB}$ & $\bigcirc$ & - & 75,5 \\
\hline $\mathrm{CQ} / 07-128$ & $\bigcirc$ & $-\mathrm{H}$ & 71,4 \\
\hline $\mathrm{CQ} / 07-129$ & $\bigcirc$ & $-\mathrm{NO}_{2}$ & 74,6 \\
\hline $\mathrm{CQ} / 07-130$ & $\bigcirc$ & $-\mathrm{CN}$ & 72,3 \\
\hline $\mathrm{CQ} / 07-131$ & $\bigcirc$ & - -sO2CH & 69,9 \\
\hline $\mathrm{CQ} / 07-133$ & $\bigcirc$ & dibenzofuran & 68,1 \\
\hline $\mathrm{CQ} / 07-134$ & $\bigcirc$ & naphthalene & 72,0 \\
\hline $\mathrm{CQ} / 07-027$ & $\bigcirc$ & anthracene & 71,6 \\
\hline
\end{tabular}

\subsection{Methods and apparatus}

To determine the conversion rate of monomers, the real time-FT-IR method was used. The equipment was composed of the Thermo Scientific i10 NicoletTM spectrometer with an appropriate horizontal adapter adapted for real time measurements of photopolymerization processes of samples with a thickness of $1.4 \mathrm{~mm}$. As the light source for photopolymerization measurements different diodes (from Thorlabs Inc.) was used. The light was emitted by the UV-LED diode to the sample with a liquid fiber optic cable (from Torlabs Inc.) with a diameter of $5 \mathrm{~mm}$ and a length of 1.2 meters was delivered [16]. Measurements were recorded in the OMNIC program.

The photocurable formulations were deposited on a PP films under air. The progress of photopolymerization process was monitored by observing the disappearance of the band at wave number equal approximately $6165 \mathrm{~cm}^{-1}$ (Figure 3 ). The UV-LED diode was started 10 seconds after the start of spectral registration. 


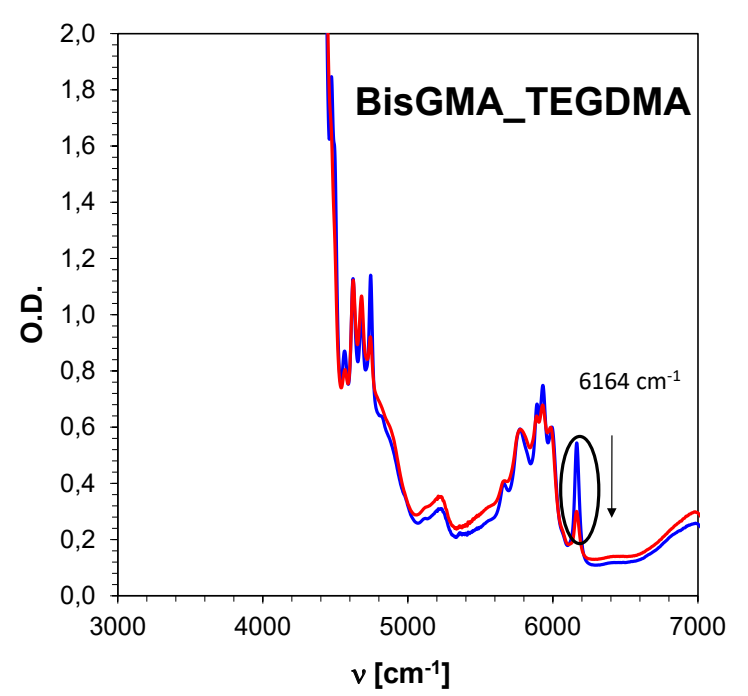

Fig. 3. Near IR spectra recorded before and after polymerization for $\mathrm{CQ} / \mathrm{EDB}$ in a molar ratio of $1: 3$ photoinitiating system and BisGMA and TEGDMA monomers followed at $6165 \mathrm{~cm}^{-1}$.

Because the decrease of absorption of the peak area is directly proportional to the number of polymerized groups, the degree of conversion of the function group was calculated by measuring the peak area at each time of the reaction by using equation (1):

$$
C_{F T-I R}[\%]=\left(1-\frac{A_{\text {After }}}{A_{\text {Before }}}\right) * 100 \% \text { (1) }
$$

where $A_{\text {Before }}$ is an area of the absorbance peak characteristic for used monomer and type of photopolymerization before polymerization process and $\mathrm{A}_{\mathrm{After}}$ is an area of the same absorbance peak, but after polymerization process.

\section{Results and discussion}

The photopolymerization process was carried out for only 100 seconds. The compositions were irradiated by a LED with maximum emission at a wavelength of $455 \mathrm{~nm}\left(18 \mathrm{~mW} / \mathrm{cm}^{2}\right)$. The samples were $1.4 \mathrm{~mm}$ thick, so they are applied in dental offices by dentists. Figure 4 shows the dependence of the conversion on time. Camphorquinone alone is not effective in initiating photopolymerization processes of BisGMA / TEGDMA monomers in a weight ratio of $7: 3$. Nevertheless, the initiation systems consist of camphorquinone and tosyl salts significantly improve the conversion rates obtained. The conversion for all initiation systems is comparable and ranges from $68,1 \%$ for the initiating system composed of compound 07-133 (having the benzofuran substituent), to as much as $74,6 \%$ for the initiating system composed of caphoroquinone and compound 07-129 (having the $-\mathrm{NO}_{2}$ substituent) (Table 1).

Moreover, the conversion and speed of the photopolymerization process are comparable to those of the CQ and aromatic amine EDB reference initiator system. The color of the obtained dental composites was also analyzed. then it was shown that only the yellowing of the composite is visible for the initiating system composed of the caphoroquinone and the tosyl salt having the nitro substituent (Table 1).

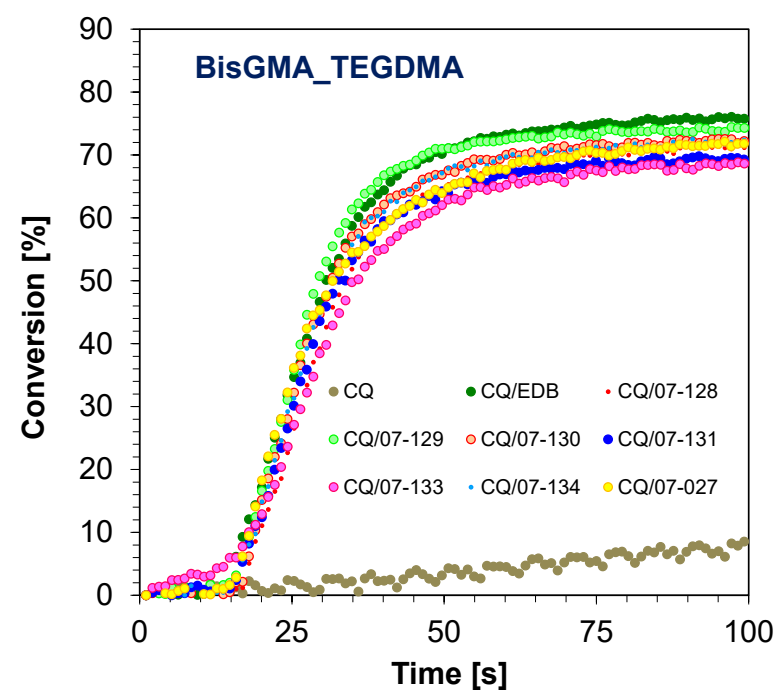

Fig. 4. Polymerization profiles (methacrylate function conversion) for BisGMA/TEGDMA in a weight ratio of 7:3 and initiating system based on CQ and different iodonium salts with tosyl anion.

\section{Conclusion}

The above communication presents alternative initiating systems based on caforoquinone and tosyl salt in a 2: 1 molar ratio to initiate photopolymerization processes of acrylate monomers used in the production of dental fillings. These systems are comparable to the standard camphorquinone and aromatic amine initiator EDB. As a result, cyto and genotic amine were eliminated from the initiating system, which is extremely beneficial in the production of new generation dental composites.

\section{Acknowledgement}

This work was financed by the Polish Ministry of Science and Higher Education from budget funds 
for science in the years 2018-2022 as a research project no. 0052/DIA/2018/47 under the "Diamond Grant" program.

\section{References}

1. C. Dietlin, S. Schweizer, P. Xiao, J. Zhang, F. Morlet-Savary, B. Graff, J.P. Fouassier, and J. Lalevée, Polym. Chem., 6(21) (2015) 3895-3912.

2. W. Funke, Prog. Org. Coat., 6 (1980), 110.

3.M. Kaur, and A. K. Srivastava, J. Macromol. Sci. Polymer Rev., 42(4) (2002) 481-512.

4. M. Layani, X. Wang and S. Magdassi, Adv. Mater, (2018), 1706344.

5. I. V. Khudyakov, J.C. Legg, M. B. Purvis, and B. J. Overton, Ind. Eng. Chem. Res., 38(9) (1999) 3353-3359.

6. E. Andrzejewska, Prog. Polym. Sci., 26(4) (2001) 605-665.

7. S. C. Ligon, B. Husár, H. Wutzel, R. Holman R. and R. Liska, Chem. Rev., 114(1) (2013) 557-589.

8. H.Y. Marghalani, H.Y. Handbook of Bioceramics and Biocomposites. Berlin/Heidelberg, Germany: 1st ed.; Springer, 2014; ISBN 9783319092300 .
9.A.A. Zandinejad, M. Atai, And A Pahlevan, Dent. Mater., 22 (2006) 382-387.

10.N.B. Cramer, Ch.L. Couch, K. M. Schreck, J. E.Boulden, R.Wydra, J.W. Stansbury and Ch. N. Bowman, Dent. Mater., 26(8) (2010) 799-806. 11. J. Ge, M. Trujillo and J. Stansbury, Dent. Mater., 21(12) (2005) 1163-1169.

12. M. Topa and J. Ortyl, Materials, 13 (2020), 4093. 13. B. Pratap, R.K. Gupta, B. Bhardwaj and M. Nag, Japanese Dental Science Review, 55(1) (2019) 126138.

14. R. Srivastava, Wolska, J. WalkowiakKulikowska, H. Koroniak and Y. Sun, Europ. Polym. J., 90, (2017) 334-343.

15. J. W. Stansbury, Journal of Esthetic and Restorative Dentistry, 12(6) (2000) 300-308.

16. M. Topa, F. Petko, M. Galek, K. Machowski, M. Pilch, P. Szymaszek and J. Ortyl, Polymers 11 (2019) 1756. 